\title{
Fundamento y Componentes de la Educación a Distancia
}

\author{
(Basis and Components of Distance Education)
}

\author{
LORENZo García ARETIO \\ Universidad Nacional de Educación a Distancia \\ (España)
}

\begin{abstract}
RESUMEN: Se presentan las posibles bases de una opción de marco teórico integrador sobre la educación a distancia. ¿Qué entendemos por teoría de la educación a distancia? Se mencionan los conocimientos que se hacen precisos para dar racionalidad científica a esta modalidad educativa (gnoseológicos, tecnológicos y axiológicos). Se esbozan las más conocidas teorías sobre educación a distancia para desembocar en la denominada teoría sobre el diálogo didáctico mediado arrancando de los diferentes tipos de diálogo que pueden establecerse en el ámbito educativo. El trabajo se culmina describiendo los diferentes componentes que conforman ese marco teórico aludido: el alumno, el docente, los materiales o soportes de los contenidos, las vías de comunicación y la infraestructura organizativa y de gestión en que se integran.
\end{abstract}

\section{Teoría - Componentes - Educación a Distancia}

ABSTRACT: The possible bases of an integrated theoretical frame for distance learning are outlined. The question of what is understood by distance education is asked. The theoretical knowledge necessary for a credible scientific basis for this subject (gnoseologic, technological, axiologic) are discussed. The most significant theories are outlined in search of a common educational discourse. The paper summarises the various aspects included in this theoretical framework; student, teacher, materials and resources, communications and organisational infrastructure in which they are integrated. 


\section{TEORIZAR SOBRE LA EDUCACIÓN A DISTANCIA}

La educación a distancia es una metodología, una modalidad, un sistema o un subsistema educativo -según el criterio clasificatorio o concepción que de ella se tenga- que, al igual que otros propósitos o propuestas de esta índole, requieren fundamentar y justificar sus virtualidades y hallazgos, así como sistematizar sus principios y normas. Es decir, con el fin de dar racionalidad científica a la educación a distancia, precisaríamos conocimientos de distinta índole:

u gnoseológica (comprensión y explicación de la realidad de la educación a distancia y de su práctica pedagógica en cuanto actividad intencional);

u tecnológica (saber hacer que permita transformar hacia mejor esa realidad educativa, proyectando, regulando, conduciendo y controlando las secuencias de intervención);

ux axiológica (desde una dimensión de valores o metas de formación que marquen el deber ser).

Elaborar ese necesario marco teórico en el ámbito de la educación a distancia, nos llevaría a observar los aspectos a los que se refiere esta realidad educativa y reflexionar sobre ellos:

o qué rasgos definen a la educación a distancia,

o cuáles son sus componentes,

o cómo son,

o cómo se relacionan entre sí,

o qué principios los sostienen,

o qué normas generan,

o cómo se aplican éstas y por qué,

o qué sucederá si se aplican de una u otra manera, etc.

No se trataría sólo de especular y reflexionar sobre el fenómeno innovador, sino de observar y comprender los hechos empíricos que muestra esta práctica educativa, como fuente para sistematizar el cómo hacer tecnológico en enseñanza a distancia y el propio qué hacer, con el fin de reelaborar los principios, leyes y normas que posibilitan una forma de enseñanza no presencial de efectos positivos. Esto llevaría a la generación de una teoría de la educación a distancia que en sentido amplio la entenderíamos como. (García Aretio, 1989: 91):

La construcción científica que consiste en la sistematización de las leyes, ideas, principios y normas, con objeto de describir, explicar, comprender y predecir el fenómeno educativo en la modalidad a distancia y regular la intervención pedagógica en este ámbito.

Por consiguiente, elaborar un marco teórico sobre la educación a distancia haría preciso manejar los saberes que se recogen en el siguiente cuadro (Medina Rubio y García Aretio, 1993) :

Uu El conocimiento riguroso del sistema conceptual y del lenguaje específico de la educación a distancia. Este conocimiento es necesario para:

o sistematizar las leyes, ideas, principios y normas que la conforman.

o garantizar un adecuado nivel de comprensión de esta modalidad de enseñanza que permita describirla y explicarla.

o entender e interpretar en su verdadero alcance los problemas que plantea la educación a distancia.

o comprender el significado de los conocimientos ya consolidados en este campo.

u La explicación y regulación del proceso de intervención pedagógica en esta modalidad que se basa en los sistemas de comunicación didáctica entre el docente y los estudiantes, a través del correspondiente diálogo, habitualmente mediado, sea éste simulado, real, síncrono o asíncrono.

El conocimiento de las técnicas, estrategias y modelos de investigación educativa que se utilizan con positivos resultados en este ámbito de conocimiento. Se hace necesario conocer los recursos pluri-metodales de análisis educativo, acordes con el marco actual de las Ciencias de la Educación, necesarios para el diseño, aplicación y

\footnotetext{
2 En el texto citado nos referíamos a la Teoría de la educación en general

${ }^{3}$. Estos autores se refieren a la Teoría de la educación, en general
} 
valoración de métodos y técnicas de investigación especializada.

Por tanto, ese marco teórico estaría basado en un aporte conceptual que delimite el objeto de esta modalidad educativa, en los procedimientos adecuados para la intervención pedagógica y en los métodos de investigación que puedan generar teorías y mejorar la práctica.

Pensamos, finalmente, que para elaborar una teoría de la educación a distancia sería preciso dar respuesta a preguntas tales como: ¿por qué la educación a distancia?, ¿para qué?, ¿qué es?, ¿cómo se hace?, ¿cuándo?, ¿dónde?, ¿a qué ritmo?, ¿quién la imparte?, ¿para quién?, ¿cuánto cuesta?...

\section{DESTACADAS TEORÍAS SOBRE LA EDUCACIÓN A DISTANCIA}

Quizás las aproximaciones más sólidas a la hora de ofrecer una perspectiva teórica del fenómeno de la educación a distancia, han sido estudios centrados en:

Uu El carácter de la forma industrial de enseñar y aprender que suponen estas propuestas (Peters, 1971 y 1993) que incluirían: planificación, división del trabajo, producción masiva, automatización, estandarización y control de calidad..

Uu El carácter autónomo e independiente de la forma de aprender ha sido destacado por Wedemeyer (1971 y 1981) centrando su planteamiento en la independencia del estudio por correspondencia no sólo con respecto al espacio y tiempo sino también en su potencialidad de independencia en el control y dirección del aprendizaje. Moore, 1977), reforzó esta teoría desde el mismo momento en que a principios de la década de los 70 conoció a Wedemeyer. Moore desarrolló una denominada teoría transaccional de la educación a distancia o "distancia transaccional" en la que sugiere dos variables críticas: diálogo (profesor-alumno) y estructura (del curso). Ambas variables en relación con la autonomía del que aprende. Mediante esta teoría pueden explicarse la naturaleza de los programas y cursos y las conductas de profesores y estudiantes.

u El énfasis en la interacción y comunicación entre docentes y estudiantes que Holmberg (1985), glosó con el término de conversación didáctica guiada que suponía una comunicación no contigua apoyada por una comunicación simulada a través de la interacción del estudiante con los materiales de estudio y una comunicación real a través de la interacción escrita y/o telefónica. Es posible que esta aportación de Holmberg haya sido la más destacada en el ámbito de la educación a distancia. La insistencia en la interacción y comunicación son también propios de Bååt (1984) y Garrison (1993).

u Lo determinante que resultan las tecnologías para la modalidad (Body, 1993; Bates, 1993; Saba, 1988 y Saba y Shearer, 1994) es otro ámbito de desarrollos teóricos más actuales.

Puede resultar de interés el amplio elenco de propuestas investigadoras que se presentan en una publicación de la UNED de España (García Aretio, 1997) en distintos estudios (26 en total) sobre dicha institución universitaria.

Todo este estado de la cuestión nos hace atrevernos a esbozar nuestro propio planteamiento teórico sobre la educación a distancia, fruto del estudio y de la propia experiencia.

\section{UNA PROPUESTA TEÓRICA INTEGRADORA}

Ya hemos analizado en otra parte (García Aretio, 1994) qué es la educación a distancia y qué rasgos la definen. Más adelante vamos a acercarnos a los componentes y estructuras de esta modalidad educativa y a través del análisis de cada uno indagar en las relaciones entre ellos. Estos componentes y sus relaciones los integraremos en lo que vendremos en denominar la estructura o espacio de la educación a distancia. Hemos mencionado los principales apuntes teóricos que centran su atención sobre algunos de los componentes de esa estructura. Parece que estaríamos en condiciones de sugerir una propuesta teórica integradora de las anteriores y lo haremos en base al gráfico que ofrecemos. Denominaremos a esta propuesta teórica, integradora o del diálogo didáctico mediado.

\subsection{LOS TIPOS DE DIÁLOGO}

Desde el punto de vista del docente, hablar de educación a distancia es hablar de una relación didáctica o diálogo con los estudiantes, que no sucede en el mismo espacio y si fuese así, no se realiza en tiempo real. Desde el punto de vista 
del alumno, se trata de una forma flexible de aprender que no exige su presencia física ni la del profesor en el mismo lugar y/o tiempo. Queremos decir, por tanto, que, por exclusión, la relación siempre la calificaremos de a distancia mientras no sea a la vez síncrona y en el mismo espacio. En definitiva y en una primera aproximación, estas propuestas de interacción o diálogo duidáctico, las resumiríamos en el siguiente cuadro.

\begin{tabular}{|c||c|c|}
\hline \multicolumn{1}{|c||}{} & \multicolumn{2}{c|}{ E S P A C I O } \\
\cline { 2 - 3 } \multicolumn{1}{|c||}{ TIEMPO } & EL MISMO & DIFERENTE \\
\hline \multirow{2}{*}{ SÍNCRONO } & $\mathbf{1}$ & $\mathbf{2}$ \\
& Presencial & A Distancia \\
\hline ASÍNCRONO & $\mathbf{3}$ & $\mathbf{4}$ \\
\hline
\end{tabular}

La relación síncrona, establecida en tiempo real, tiene indudables ventajas de carácter interactivo. Sin embargo, la asincronía, que no requiere la participación simultánea de profesores y estudiantes, manifiesta otras ventajas, tales como las de permitir la flexibilidad de elección de contenidos, tiempo de estudio, apoyos alternativos, relación con otros estudiantes, etc.

En el cuadrante 1 inscribiríamos a toda aquella relación educativa que se produce cuando educador y educando pueden verse y oirse físicamente, sin mediación alguna. Tradicionalmente parecía reservarse el cuadrante 4 para lo que se denominaba educación por correspondencia y después, a distancia. Hoy nadie pone en duda que los espacios 2 y 3 han de reservarse también a estas formas alternativas de enseñar/aprender que nosotros denominamos genéricamente como formas de enseñanza y aprendizaje abiertos y a distancia.

Partimos, por tanto, de un diálogo didáctico (comunicación didáctica) de doble vía entre dos entes separados físicamente uno de otro, sea en el espacio, en el tiempo o en ambos a la vez, y establecido a través de unos medios conformados por materiales preproducidos y por unas vías de comunicación sea esta comunicación síncrona o asíncrona. Ese diálogo o interacción entre el que enseña y el que aprende se convierte, obviamente, en el elemento central de cualquier proceso de enseñanza-aprendizaje

\subsection{EL DIÁLOGO SIMULADO}

La institución u organización es la que enseña [organización que ayuda le llama Delling (1987) y organización de apoyo le denomina Holmberg (1989)], no el profesor. En la enseñanza presencial es el docente el que habitualmente diseña, produce, distribuye, desarrolla y evalúa el proceso de enseñanza-aprendizaje, por lo que el aprendizaje del alumno suele estar en función de que le haya correspondido un buen o un mal docente. En la enseñanza a distancia, sin embargo, el docente nunca es uno, son multitud los agentes que intervienen en el proceso de enseñar y aprender, hasta tal punto que solemos reconocer a la institución como la portadora de la responsabilidad de enseñar. Por tanto, es la institución, más que el profesor, la que diseña, produce, distribuye, desarrolla o tutela el proceso de aprendizaje de los estudiantes.

El interés de una institución que enseña es el de que se produzca aprendizaje en el receptor del proceso. Pero este aprendizaje ha de permitir al estudiante ser protagonista en cuanto al tiempo, espacio y ritmo de aprender, es decir, el proceso de enseñanza diseñado por la institución propicia el aprendizaje flexible del estudiante. Y esa flexibilidad es facilitada a través de la comunicación o diálogo didáctico mediado entre institución y estudiante. Son, en efecto, los medios los que permiten la flexibilidad antes referida.

Continuando con nuestro gráfico, nos encontramos con que es la institución la que diseña y produce los materiales para el aprendizaje. En esos materiales es donde se almacenan o empaquetan los contenidos, es en los que se concretan, mediante la metodología adecuada, los conocimientos, competencias y actitudes que se pretende que alcance el estudiante, siempre adaptados a sus necesidades y a las características de la materia o curso. Este almacenamiento se realiza en materiales impresos, audio (radio), vídeo (tv), informáticos y en el soporte web de internet. Una vez producidos estos materiales, siguiendo criterios rigurosos de planificación, se procede a la distribución de los mismos con el fin de hacerlos llegar a los destinatarios finales, los estudiantes.. 
A través de un diálogo simulado y asíncrono se establece la primera comunicación de doble vía entre la institución que enseña a través de los materiales y el estudiante que pretende aprender. En principio, unos materiales ideados para la enseñanza a distancia, deben representar algún tipo de diálogo con el destinatario de los mensajes. Pero será un diálogo simulado, nunca real, y por la propia naturaleza del soporte, de carácter asíncrono. No pueden coincidir en el tiempo la producción o emisión del mensaje, con la respuesta del receptor.

Pues bien, estos materiales una vez distribuidos son estudiados por el alumno de forma relativamente independiente y autónoma. Es el estudiante el que decide si desea estudiar o no y cuándo hacerlo. Decidirá también sobre el ritmo y pasos que habrá de seguir, los objetivos y medios, la forma de cómo estudiar. Un estudiante de enseñanza a distancia tiene un amplio campo de independencia contrariamente a lo que sucede con la dependencia o supeditación al profesor, propia de un estudiante presencial.

\subsection{EL DIÁLOGO REAL}

Pero este diálogo mediado a través de los materiales sería insuficiente si no se dispusiera de las adecuadas vías o canales de comunicación, permanentemente abiertos y a disposición de los alumnos, que permitiesen un diálogo real y no simulado. Un diálogo donde el estudiante no se limite a responder, sino a preguntar o a iniciar el mismo. Este diálogo real toma cuerpo a través de una enseñanza tutelada. En efecto, la tutoría es un elemento sustancial y singular de los sistemas a distancia. Suele ser el rostro, la imagen de esta forma de enseñar-aprender al establecer un sentimiento de relación personal entre el que enseña y el que aprende. Este diálogo tutorial se viene estableciendo a través de las vías: presencial, postal, telefónica, fax, videoconferencia de sala, internet (correo electrónico, noticias, listas, www, videoconferencia, etc.). En la mayoría de instituciones y programas a distancia, el componente de estudio independiente o autónomo está invariablemente apoyado por sistemas de asistencia y asesoramiento que son proporcionados idealmente en centros de estudio o de apoyo o, en mayor medida, por medios modernos de comunicación.

Esta segunda parte de diálogo mediado real puede producirse de forma síncrona (presencial, telefónica, videoconferencia, internet) o asíncrona (postal, fax, internet). En definitiva, este diálogo didáctico mediado pretende producir un aprendizaje pero no en solitario sino guiado.

En fin, la teoría del diálogo didáctico mediado, basa su propuesta en el diálogo a través de los medios que, cuando se trata de los materiales, descansa en el autoestudio y cuando se trata de las vías de comunicación, en el refuerzo que desde la institución, a través de la tutoría se hace para el logro de los objetivos de aprendizaje. Realmente la base de la educación a distancia, su rasgo más destacado al diferenciarla de la modalidad presencial, es su característica de comunicación mediada entre docentes y alumnos (Garridson, 1993). Así entendemos que la enseñanza a distancia se basa en un diálogo didáctico mediado entre el profesor u organización que tutelan y el estudiante que, separado físicamente de aquél, aprende de forma independiente y flexible.

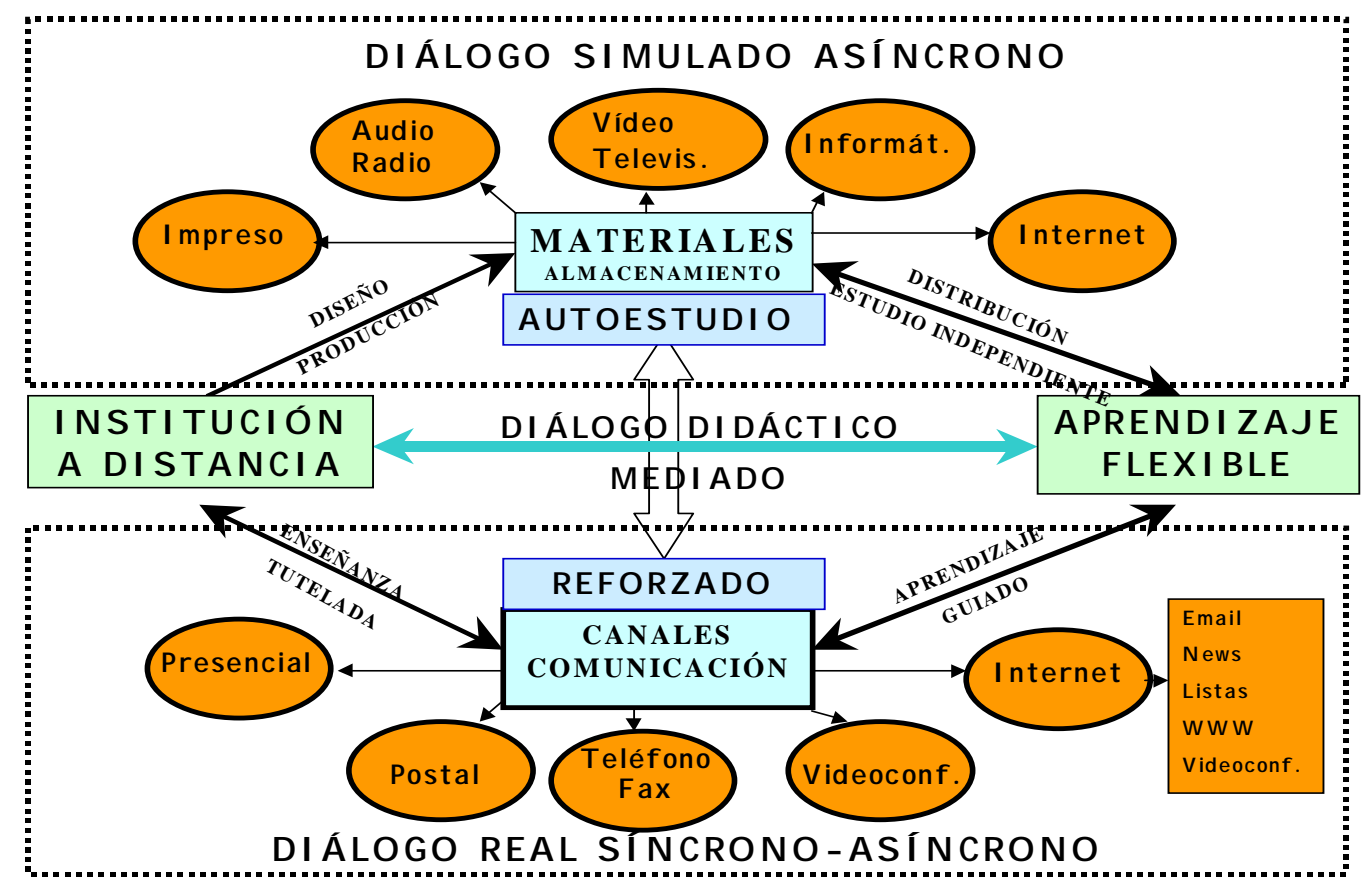




\section{COMPONENTES DEL SISTEMA DE EDUCACIÓN A DISTANCIA}

Esta propuesta teórica la podemos cerrar considerando los componentes que interaccionan en esa realidad educativa en la que se ha de aplicar la metodología de enseñanza/aprendizaje más adecuada, de acuerdo con:

ue tipo de alumnos destinatarios de la formación;

$u$ el tipo o modalidad de comunicación didáctica de que se puede disponer dentro de la norma o modelo educativo propuesto;

el tipo de estructura organizativa en el que se integra su docencia, y

u el tipo de tareas docentes que expresamente se le requieren dentro de la modalidad educativa diseñada.

Por todo ello, nos referiremos a los componentes o elementos básicos que se integran en el sistema a distancia y cuyas características y/o funciones se diferencian sustancialmente de las de las análogas de los sistemas convencionales y que son: el alumno, el docente, los materiales o soportes de los contenidos, las vías de comunicación y la infraestructura organizativa y de gestión en que se integran (García Aretio, 1994). A estos componentes clásicos podemos agregar otros que vienen siendo también comunes a otras instituciones de enseñanza convencional. Con todos ellos nos atrevemos a construir el espacio o estructura de la educación a distancia.

\subsection{EL ALUMNO}

El elemento básico en todo hacer educativo es el destinatario del mismo y en función del que se estructura todo el proceso, el alumno. Conocer su desarrollo psicológico, estilos de aprendizaje, motivaciones, etc., es imprescindible para el buen desempeño de la acción de educar.

Los sistemas de enseñanza a distancia se han establecido generalmente para atender a una población adulta que aprende y se manifiesta de manera diferente al niño, adolescente o joven, habituales usuarios de las realizaciones convencionales. En contraposición al de estas instituciones educativas, el alumno a distancia es un individuo generalmente maduro con una historia vivencial llena de experiencias, conocimientos, capacidades, hábitos, actitudes, conductas e interés en participar en su propio proceso de formación, características éstas que condicionan, filtran $\mathrm{y}$, previsiblemente, mejoran los futuros aprendizajes.

Obviamente la metodología para motivar y enseñar a estos individuos ha de ser diferenciada de la utilizada en la enseñanza convencional, porque este alumno de características específicas de adulto que trata de aprender, ha de hacerlo a distancia, por lo que, de acuerdo con lo ya expuesto, habrá de recorrer la mayor parte del proceso de forma autónoma e independiente.

Esto potenciará, precisamente, su capacidad para aprender por sí mismo y su autonomía a la hora de marcarse metas a largo, medio y corto plazo. Estas metas pueden hacer referencia a:

Uu la consecución y priorización de objetivos de aprendizaje;

u la secuenciación de los contenidos;

u la selección de recursos adecuados a objetivos y contenidos;

u la programación del cuándo y cómo de la evaluación;

u la adecuación de los aprendizajes a su tiempo, ritmo y estilo de aprender.

Además de obligar a ese aprendizaje en solitario y autónomo, que a la vez propiciará la independencia y autodidactismo en futuras adquisiciones, el estudio a distancia habrá de ser individualizado y personalizado.

Lo ideal es que se adapte a las necesidades, características e intereses personales de los alumnos y a su disponibilidad de tiempo, espacio, motivaciones, ritmos y estilos de aprender, por lo que el currículo a cubrir habría de ser flexible y adaptable a estas circunstancias.

Por otra parte, no olvidemos que, aunque la población adulta sea la principal destinataria de las acciones formativas a distancia, esta modalidad no sólo atiende a estos alumnos adultos.

Se están impartiendo enseñanzas a distancia a estudiantes en el grupo de edad preuniversitario. Algunas instituciones ofrecen tiempo de aprendizaje a distancia, aprovechando las posibilidades que brindan las nuevas tecnologías. En otros 
casos se ofrecen módulos para enriquecimiento del propio plan de estudios y que pueden abordarse de forma independiente. Por otra parte alumnos jóvenes, universitarios, se están aproximando a los estudios impartidos por centros superiores que disponen de enseñanzas o programas a distancia, bien sea por problemas de residencia -en su localidad de residencia no se imparten esos estudios-, económicos o laborales.

Otro grupo de alumnos destinatarios de los programas a distancia son aquellos que sufren algún tipo de discapacidad o minusvalía física. También aquellas personas de considerable edad que no pueden moverse con facilidad pero que desean aprender más. La incorporación de las nuevas tecnologías de la información viene a suplir las desventajas iniciales básicas de las personas con discapacidad y contribuyen a "normalizar" la actividad de las mismas, equiparándola en lo sustancial a la del resto de las personas. Se rompen así las barreras que dificultan el acceso de estos ciudadanos a la obtención de determinado tipo de información.

También puede atenderse a través de este medio a personas que hablan otra lengua. Por una parte, podrían recibir formación a distancia dentro del sistema educativo de su país. También, desde el actual lugar de residencia se pueden ofrecer cursos, primero de lengua, a estos inmigrantes que, posteriormente podrían acceder a otros saberes ya en la lengua del país de su residencia actual.

\subsection{EL DOCENTE}

La eficacia y eficiencia de las instituciones educativas depende en gran parte de la formación, capacidades y actitudes de sus docentes. Esas tres características estarán en consonancia de las funciones encomendadas a los mismos que, obviamente, sabemos que son distintas en una institución a distancia respecto a otra de carácter presencial.

En la institución a distancia la docencia no es directa, se utilizan recursos técnicos más o menos sofísticados para posibilitar la comunicación bidireccional en la que colabora un profesor menos típico en las otras instituciones, el tutor. Docencia que habrá de ser enfocada a motivar y potenciar el aprendizaje independiente y autónomo de un adulto. Y, por fin, se requiere un proceso tecnológico, sobre todo en lo referente a la planificación previa, mucho más depurado que en las instituciones educativas de carácter presencial.

Estas características se convierten en condicionantes de la acción docente a distancia que aparece compleja por la multitud de agentes que intervienen desde el diseño de los cursos hasta la evaluación de los aprendizajes de los alumnos.

Por ello, las funciones sustanciales que un profesor de la enseñanza convencional desempeña de forma generalmente individual (programación, enseñanza directa y evaluación) precisan en el caso de la docencia a distancia de la intervención de equipos de expertos en los distintos campos que habrán de dividir el trabajo:

Uu Expertos en los contenidos de la disciplina o curso en cuestión.

u Especialistas en la producción de materiales didácticos: tecnólogos en educación (diseño y estructura de los contenidos), editores, diseñadores gráficos, expertos en comunicación y medios técnicos (producción transmisión de materiales audiovisuales e informáticos), etc.

u Responsables de guiar el aprendizaje concreto de los alumnos que planifican y coordinan las diversas acciones docentes (a distancia y presenciales), integran los distintos materiales, y diseñan el nivel de exigencia y las actividades de aprendizaje precisas para superar el grado de logro previsto.

u Tutores, asesores, consejeros, animadores que motivan el aprendizaje y aclaran y resuelven las dudas y problemas surgidos en el estudio de los alumnos y, en su caso, evalúan los aprendizajes.

La división del trabajo en esta modalidad de enseñanza se hace aconsejable por la propia complejidad de las funciones. Deben aprovecharse las ventajas que propician las siguientes situaciones: un buen experto en contenidos científicos pone su saber a disposición de los especialistas en elaborar un material impreso de calidad científica y pedagógica adaptado a las necesidades de un alumno que ha de estudiar a distancia; un docente formado en diseño y coordinación del proceso de aprendizaje de los alumnos y un tutor que ayuda a aprender y motiva a los alumnos. Este equipo, asegura un mayor grado de objetividad en los conocimientos objeto de aprendizaje.

En muchas instituciones, las tres primeras funciones reflejadas anteriormente son desempeñadas por la misma persona, con el riesgo que esto comporta, sobre todo respecto a la función segunda, de elaborar unos materiales faltos de calidad pedagógica. También, en función de la dimensión de la institución y de su número de alumnos, las funciones 3 y 4 pueden recaer o no sobre la misma persona. 
Nos parece positivo sin embargo, que, siempre que sea posible, las funciones 1 y 3 sean desempeñadas por el mismo docente con el fin de posibilitar un mayor grado de coordinación, que se hace fundamental cuando es la misma persona la que marca el nivel científico de los contenidos y coordina el proceso de aprendizaje de los alumnos. Así este docente sería:

u el autor de la producción científica;

$u$ el diseñador del proceso de aprendizaje y de la utilización de los paquetes instruccionales;

u el responsable de la evaluación de los aprendizajes;

u el coordinador de los tutores de su materia o curso.

Con la puesta a disposición de los alumnos de materiales didácticos de calidad queda cubierta una importante parte de la misión que la sociedad encomienda a las instituciones educativas, la de transmitir la ciencia, la técnica y la cultura. Pero será posible llevar a cabo otras funciones tales como la motivación al estudio, la crítica de los contenidos transmitidos, la aplicación de estos conocimientos, etc.?.

En un sistema a distancia estas posibilidades se propician básicamente a través de la orientación personal, la ayuda individualizada y el contacto con los compañeros que se vehiculan a través de la tutoría. Volveremos en otra próxima unidad sobre la figura del tutor, característica de este tipo de enseñanza.

\subsection{LA COMUNICACIÓN A TRAVÉS DE LOS MEDIOS}

En educación es el docente el que gobierna o debe gobernar y regular el proceso mediante el que se desarrollan una serie de acciones recíprocas entre educador y educando de manera que la acción de cada uno de ellos determina la respuesta del otro. La intencionalidad educativa del docente o de la institución que educa debe marcar la dirección del proceso interactivo. La interactividad, cuando los educandos son más de uno, además de producirse en sentido vertical se desarrolla enriquecedoramente en sentido horizontal y en múltiples direcciones dentro del grupo. En educación a distancia el estudiante interacciona real o virtualmente:

u con los docentes (profesores principales, tutores, consejeros, animadores..., que apoyan, motivan y orientan el aprendizaje)

u con los propios compañeros entre sí (alumno-alumno, alumno-grupo, con o sin el docente)

u con los materiales de aprendizaje (leyendo, viendo, escuchando, manipulando, seleccionando, interpretando, asimilando, sintetizando...).

u con la institución (sede central o centros de apoyo) con el fin de recabar servicios administrativos y resolver problemas de tipo general.

Todas estas posibilidades interactivas, en educación a distancia pueden adoptar diversas modalidades, en función de la intermediación, del tiempo y del canal.

\subsubsection{En función de la intermediación:}

uresencial, que es la interacción cara a cara, que a la vez es síncrona y real; parece la forma ideal de interactividad dado que no existe ningua intermediación entre los sujetos que se relacionan.

Uu no presencial o mediada a través de algún material o canal de comunicación; toda la interactividad que no se produce de forma directa, cara a cara, entraría en esta categoría.

\subsubsection{En función del tiempo puede ser:}

u síncrona, cuando tiene lugar en tiempo real simultáneo e inmediato a la producción del mensaje (ej.: conversación presencial, telefónica, videoconferencia, charla interactiva...), y

$u$ asíncrona, en la que la relación no se produce en tiempo real, la emisión del mensaje, la recepción y la posible nueva respuesta (realimentación) al mismo no se producen de forma simultánea sino diferida en el tiempo, durante minutos, horas o días (ej.: el correo postal o electrónico).

\subsubsection{En función del canal:}


u real, la que objetivamente se produce sea de forma síncrona o asíncrona mediante un canal o vía de comunicación (ej.: presencial, postal, telefónica, teleconferencia, telemática, etc.), y

uv virtual, en la que se produce un tipo de diálogo irreal, imaginario o virtual entre el autor del material (impreso, audiovisual o informático) y el usuario; realmente se interactúa con el propio material, siendo el caso más significativo la interacción con productos multimedia o vías como internet.

Sería interactividad auténtica (Bretz, 1983) aquella en la que el emisor y receptor intervienen alternativamente invirtiendo sus papeles en cada turno de palabra. Esta interactividad sólo sería posible en cada una de las relaciones que hemos definido como presencial, síncrona y real. Las otras serían, en términos del mismo autor, cuasi-interacción (interacción más frecuente en los diálogos hombre-máquina) e interactividad simulada. Una forma resumida de todos los tipos de interactividad la podemos presentar así:

\begin{tabular}{|l||l|l|l|}
\hline \multicolumn{1}{|c||}{ TIPOS } & \multicolumn{2}{c|}{ VIRTUAL } \\
\cline { 2 - 4 } & Síncrona & Asíncrona & \\
\hline \multirow{2}{*}{ Presencial } & Invividual y & \multicolumn{1}{c|}{ X } & \multicolumn{1}{c|}{ X } \\
\hline Grupal & $\begin{array}{l}\text { Teléfono, } \\
\text { Videoconferencia y } \\
\text { WWW }\end{array}$ & $\begin{array}{l}\text { Correspondencia y } \\
\text { Telemática (E-mail, } \\
\text { News, Listas de correo, } \\
\text { WWW...) }\end{array}$ & $\begin{array}{l}\text { Mat. Impreso, } \\
\text { Mat. Audiovisual, } \\
\text { Mat. Informático, } \\
\text { WWW... }\end{array}$ \\
\hline
\end{tabular}

Sin comunicación no sería posible la educación dado que en ésta alguien (emisor) pone a disposición de otros (receptores) un mensaje educativo a través de un canal que permitirá recibir el mensaje simultáneo a su emisión o diferido. El correspondiente feed-back completará el circuito comunicativo convirtiendo al receptor en emisor y vuelta a empezar el proceso.

Esta comunicación completa, bidireccional, es la requerida en educación, y si entendemos que a distancia puede educarse, también en este caso se hace necesaria. Los avances técnicos ponen a disposición de los protagonistas implicados en el proceso de comunicación (profesores y alumnos) una serie de medios que posibilitan diferir en espacio y/o tiempo la emisión y recepción de los mensajes pedagógicos con el fin de salvar precisamente esos obstáculos tempoespaciales. Éste es el rasgo diferencial más definitivo de la educación a distancia, el de la comunicación mediada entre profesores y alumnos (Garridson, 1993).

Uno de los problemas que más acusan los alumnos de esta modalidad de enseñanza es el de la soledad y alejamiento del profesor y de los compañeros de estudio. La necesidad de relacionarse con los otros se convierte a veces en determinante para el logro de resultados de aprendizaje. Pues bien, ahí está el reto de la enseñanza a distancia. En ella, prescindiendo de la habitual presencia cara a cara de profesor y alumnos, se pueda mantener un eficaz sistema de comunicación no presencial. Si ello es así, el alumno no se sentirá tan solo y, por el contrario, contará con la orientación y motivación del profesor y, en su caso, de los propios compañeros.

Pero tengamos en cuenta que, mientras en la enseñanza presencial se realiza el diseño instructivo de una materia o curso y posteriormente se planifican los medios o recursos que se van a emplear para la eficacia del proceso de enseñanzaaprendizaje, en la enseñanza a distancia los medios o recursos que se pueden utilizar pueden estar condicionando desde el principio el propio diseño de la docencia (es el caso de la enseñanza por radio que en España desarrolla Radio ECCA).

Por tanto, habrá de contarse primero en qué modalidad de comunicación (personal directa, impresa, audiovisual, informática, etc.) va a basar la institución el proceso de enseñanza-aprendizaje que pretende aplicar o cuál de ellas va a primar sobre las demás para, en función de ello, organizar y planificar el diseño instructivo que integre todos los recursos utilizables de manera que se adapten al alumno y a sus singularidades de aprendizaje. 
El sistema de comunicación habrá de apoyarse en los canales o medios que soporten esa comunicación. Los primeros sistemas por correspondencia utilizaban casi exclusivamente el material impreso vía postal. Los actuales sistemas a distancia utilizan:

\section{Materiales:}

- Impresos enviados por correo (unidades didácticas, módulos de aprendizaje abierto, guías de curso, guías de orientación didáctica, cuadernos o módulos de evaluación, addendas de ampliación o complemento, circulares, etc.) o por telefax, soporte que se está manifestando como vehículo ideal para envíos puntuales y de reducida extensión.

o Audiovisuales (cassette, video, radio, televisión...).

o Informáticos (programas informáticos específicos, CD-ROM, videodisco interactivo, hipermedia, Internet...).

\section{u Vías de comunicación:}

o Tradicionales (presencial, postal y telefónica).

o Videoconferencia e Internet (Email, news, listas, www...)

Todos estos elementos deben conformarse como sistema multimedia, integrados de forma que cada recurso cumpla su función en el momento adecuado del proceso de aprendizaje y siempre formando unidad con el resto de los medios.

A estas formas de establecer comunicación se les exige en un buen diseño de educación a distancia el que sean bidireccionales posibilitando tanto el que el alumno responda a los trabajos o cuestiones planteados en el material de estudio como a que plantee a los profesores que dirigen el proceso o a sus tutores (mediante el teléfono, la correspondencia, el ordenador o por vía presencial) las cuestiones que estime pertinente y que mejoren la eficacia de su estudio.

\subsection{ESTRUCTURA, ORGANIZACIÓN Y GESTIÓN}

Destaquemos en este componente sólo los aspectos que distinguirían la modalidad a distancia de la enseñanza presencial. Es decir, cualquier institución educativa deberá contar con una unidad o sección de información y matrícula o inscripción de los alumnos, sea esta convencional o a distancia. Sin embargo, una institución de carácter presencial no tiene que contar, por ejemplo, con centros de apoyo al aprendizaje de los alumnos, exteriores a la sede central o centro de recursos. En consecuencia, una institución de enseñanza a distancia deberá disponer de las siguientes unidades y funciones:

Uu Unidad o sección de diseño y producción de materiales que habrá de contar con los expertos en contenidos y en diseño del tipo de material de que se trate.

un Unidad de distribución de materiales con la función de hacer llegar éstos de forma puntual a sus destinatarios dispersos geográficamente.

uroceso de comunicación que precisa de una atención específica en estas instituciones con el fin de coordinar y garantizar el funcionamiento de los más diversos medios que posibiliten la comunicación bidireccional (correo, teléfono, radio, tv, telemática...).

u La coordinación del proceso de conducción del aprendizaje se hace precisa habida cuenta de la diversidad de agentes que intervienen en el mismo: productores de materiales, responsables del proceso de enseñanza-aprendizaje, tutores y, en su caso, evaluadores.

u La evaluación a distancia y/o presencial comporta una estrategia también diferente a la de la enseñanza presencial por lo que habrán de arbitrarse las instancias precisas para su adecuado funcionamiento. Además se hace necesaria para realimentar el propio sistema en aras de una mejor calidad de los procesos y, consecuentemente, de los productos.

En todo caso, la producción y distribución de materiales, podrían ser externos a la institución.

Por fin, algo característico de este tipo de instituciones, son los centros o unidades de apoyo o de estudio dispersos por el país o región donde tiene sus influencias la institución de educación a distancia, estando condicionado su cantidad e incluso su existencia a la extensión de la zona geográfica de acción y al número de alumnos atendidos. Estos centros pueden ser 
autónomos en cuanto a su funcionamiento económico-administrativo, aunque dependientes en el plano académico de la sede central, o dependientes plenamente de la institución central en todos sus aspectos. En estos centros el alumno puede:

u Disponer de edificios y equipamientos tales como aulas, biblioteca, mediateca, laboratorio, etc.

u Informarse y resolver sus problemas de orden burocrático tales como inscripción, adquisición de materiales, envío y recepción de materiales de evaluación a distancia.

u Conectar con el tutor -a través de la tutoría- con el fin de recibir la orientación pertinente que le permita aclarar dudas, integrar los distintos materiales de estudio, reforzar sus aprendizajes.

Ue Relacionarse con los compañeros que están en situación similar en cuanto a problemas y circunstancias.

Uu Usar del nexo que le permite estar "más cerca" de la sede central.

En determinados casos, estos centros pueden convertirse en foco cultural de la zona geográfica de influencia.

Una buena administración y un adecuado sistema de dirección precisan además de un personal eficiente y cualificado, unos eficaces diseños, sistemas administrativos modernos, sistemas de planificación y seguimiento, organización presupuestaria y contable eficiente, etc., que sin duda mantienen diferencias con los propios de las instituciones presenciales (Unesco, 1998).

\subsection{OTROS COMPONENTES MENOS ESPECÍFICOS}

De acuerdo con el documento UNESCO (1998), a los citados podríamos agregar:

u La misión. En un sistema de educación a distancia, la misión define el rol del sistema dentro de un contexto concreto de política educativa. Puede dirigirse hacia objetivos concretos, grupos diana, regiones, sectores o niveles de enseñanza, y guiados por valores y filosofías específicas de la enseñanza.

u Los programas y currículos. Éstos definen el perfil de un sistema o una institución. Deberán estar relacionados con la misión y los mercados y necesidades específicas.

uas técnicas y estrategias de enseñanza. Dependen en parte del tipo de programa y de las necesidades que se pretende satisfacer. También dependen de la filosofía y valores educativos del sistema escogido, y del potencial y características educativas de la tecnología utilizadas.

u Relación entre los alumnos. En bastantes sistemas a distancia esta relación es inexistente. Sin embargo, se suele considerar importante. Como no siempre es posible la reunión personal de grupos de alumnos, puede suplirse a base de organización de grupos virtuales a través de las tecnologías.

Como puede comprobarse, en el marco teórico que hemos esbozado destaca la relación mediada entre docente y alumnos. Es esta la característica que más diferencia un programa o acción formativa presencial, en el que la relación habitualmente es directa, de otro a distancia.

\section{REFERENCIAS BIBLIOGRÁFICAS}

BÅÅTH, J.A. (1984). Pride and prejudice among Distance Educators. ICDE. Bulletin, Vol. 5, May.

BATES, T. (1993). Theory and practice in the use of technology in distance education. En Keegan (ed) Teoretical principles of distance education. London \& New York: Routledge.

Body, G. (1993). A theory of distance education for the cyberspace era. En Keegan (ed) Teoretical principles of distance education. London \& New York: Routledge.

BRETZ, R. (1983). Media for interactive communication. London: Sage.

DELLING, R.M. (1987). Towards a theory of distance education. ICDE Boulletin, 13, pp. 21-25.

GARCía ARETIO, L. (1989). La educación. Teorías y conceptos. Madrid: Paraninfo.

GARCíA ARETIO, L. (1994). Educación a distancia hoy. Madrid: UNED.

GARCÍA ARETIO, L. (coord.) (1997). Investigar para mejorar la calidad de la Universidad. Madrid: UNED.

GARCÍA ARETIO, L. Y MARÍN, R. (coords) (1998). Aprendizaje abierto y a distancia. Perspectivas y consideraciones políticas. Madrid: UNED-UNESCO. 
GARRIDSON, D.R. (1993). Quality and access in distance education: theoretical considerations. En Keegan, D. (ed.) Theoretical principles of distance education. London: Routledge.

GARRISON, D.R. (1993). Quality and access in distance education: theoretical considerations. En Keegan (ed) Teoretical principles of distance education. London \& New York: Routledge.

HOLMBERG, B. (1985). Educación a distancia: situación y perspectivas, (traducción de 1981, Londres). Buenos Aires: Kapelusz.

HOLMBERG, B. (1989). Theory and practice of distance education. New York: Toutledge.

KEEGAN, D.J. (1983). Six Distance Education Theorists. Hagen: ZIFF.

MEDina RuBio, R. y GARCía ARETIO, L. (1993). Teoría de la educación. Guía didáctica. Madrid: UNED.

MoORE, M.G. (1977). On a Theory of independent study. En Epistolodidaktika, 1977.

PETERS, O. (1971). "Theoretical aspects of correspondence instruction". En McKenzie y Christensen. The changing world of correspondence study. Pensylvania State University Presss.

PETERS, O. (1983). Distance teaching and industrial production: a comparative interpretation. Sewart; Keegan y Holmberg (Eds). Distance education. International perspectives. London: Croom Helm.

PETERS, O. (1993). Distance education in a post-industrial society. En Keegan (ed) Teoretical principles of distance education. London \& New York: Routledge.

RumBLE, G. (1986). The Planning and Management of Distance Education. London: Croom Helm.

SABA, F. (1988). Integrated telecommunications systems and instrucctional transaction. American Journal Distance Education, 2:3, 17-24.

SABA, F. Y SHEARER, R. (1994). Verifyng theoretical concepts in a dynamic model of distance rducation. Americanm Journal Distance Education, 8 (1), $36-59$.

UNESCO (1998). Aprendizaje abierto y a distancia. Perspectivas y consideraciones políticas. Madrid: UNED-UNESCO.

WeDEMEYER, C.A. (1971). Independent study. En Deighton, L.C. (ed) The Encyclopedia of Education, 4. New York: Macmillan.

WEDEMEYER, C. A. (1981). Learning at the Back Door. Reflections on Non-Traditional Learning in the Lifespan. Madison: University of Wisconsin Press.

PERFIL ACADÉMICO Y PROFESIONAL DEL AUTOR

Lorenzo García Aretio es Director del Master de enseñanza y Aprendizaje Abiertos y a Distancia de la UNED de España. Es doctor y profesor titular de Teoría de la Educación en la Facultad de educación. Ha sido también Director del Instituto Universitario de Educación a Distancia, Coordinador general de Planes de Estudio y Director Adjuntos del Programa de Formación del Profesorado de la UNED.

Su línea de investigación se viene centrando desde hace bastantes años en todo lo relativo a la enseñanza/aprendizaje abiertos y a distancia. Como autor principal, coordinador o editor, ha publicado 13 libros relativos al tema de la educación a distancia y más de 40 artículos en libros y revistas especializados. Igualmente viene participando en numerosos proyectos financiados por la Unión Europea, siempre relativos al mismo campo.

\footnotetext{
Lorenzo García Aretio

Facultad de Educación

UNED

Senda del Rey s/n

28040 - Madrid (España)

Telf: + 34913987218

Fax: + 34913986918

Correo-E: 1garetio@edu.uned.es.
} 